\title{
Adiabaticity and gravity theory independent conservation laws for cosmological perturbations
}

\author{
Antonio Romano* \\ Universidad de Antioquia (CO) \\ E-mail: antonio.enea.romanodcern.ch
}

\begin{abstract}
We carefully study the implications of adiabaticity for the behavior of cosmological perturbations. There are essentially three similar but different definitions of non-adiabaticity: one is appropriate for a thermodynamic fluid $\delta P_{\text {nad }}$, another is for a general matter field $\delta P_{c, n a d}$, and the last one is valid only on superhorizon scales. The first two definitions coincide if $c_{s}^{2}=c_{w}^{2}$ where $c_{s}$ is the propagation speed of the perturbation, while $c_{w}^{2}=\dot{P} / \dot{\rho}$. Assuming the adiabaticity in the general sense, $\delta P_{c, \text { nad }}=0$, we derive a relation between the lapse function in the comoving slicing $A_{c}$ and $\delta P_{\text {nad }}$ valid for arbitrary matter field in any theory of gravity, by using only momentum conservation. The relation implies that as long as $c_{s} \neq c_{w}$, the uniform density, comoving and the proper-time slicings coincide approximately for any gravity theory and for any matter field if $\delta P_{\text {nad }}=0$ approximately. In the case of general relativity this gives the equivalence between the comoving curvature perturbation $\mathscr{R}_{c}$ and the uniform density curvature perturbation $\zeta$ on superhorizon scales, and their conservation. This is realized on superhorizon scales in standard slow-roll inflation.

We then consider an example in which $c_{w}=c_{s}$, where $\delta P_{\text {nad }}=\delta P_{c, \text { nad }}=0$ exactly, but the equivalence between $\mathscr{R}_{c}$ and $\zeta$ no longer holds. Namely we consider the so-called ultra slow-roll inflation. In this case both $\mathscr{R}_{c}$ and $\zeta$ are not conserved. In particular, as for $\zeta$, we find that it is crucial to take into account the next-to-leading order term in $\zeta$ 's spatial gradient expansion to show its non-conservation, even on superhorizon scales. This is an example of the fact that adiabaticity (in the thermodynamic sense) is not always enough to ensure the conservation of $\mathscr{R}_{c}$ or $\zeta$.
\end{abstract}

The European Physical Society Conference on High Energy Physics

5-12 July

Venice, Italy

* Speaker. 


\section{Adiabaticity: several definitions}

Let us consider several definitions of (non)-adiabaticity. Adiabaticity is apparently a term from thermodynamics. Therefore originally it is meaningful only when the basic matter variables such as the energy density and pressure are thermodynamic. As can be seen from the perturbed energy and momentum conservation equations for a perfect fluid with equation of state $P=P(\rho)$, adiabatic perturbations move with the speed of sound $c_{w}$, given by

$$
c_{w}^{2} \equiv \frac{P^{\prime}}{\rho^{\prime}} .
$$

For a perfect adiabatic fluid, we therefore have $\delta P=c_{w}^{2} \delta \rho$. Then it seems natural to define the non-adiabatic pressure as

$$
\delta P_{\text {nad }} \equiv \delta P-c_{w}^{2} \delta \rho,
$$

which is gauge invariant and vanishes for a perfect fluid. This is the definition used in $[4,5]$, and in much of the literature.

However, the early universe is for sure not in thermal equilibrium, so one can question the above definition based on thermodynamics. In fact, when the universe is dominated by a scalar field, it makes more sense to talk about the propagation speed $c_{s}$ of that scalar field (the phase speed of sound, see also [6]), defined on comoving slices via

$$
c_{s}^{2} \equiv\left(\frac{\delta P}{\delta \rho}\right)_{c} .
$$

One is then led to define the non-adiabatic pressure as

$$
\delta P_{c, \text { nad }} \equiv \delta P_{c}-c_{s}^{2} \delta \rho_{c} .
$$

For a fluid, one has $c_{s}=c_{w}$ and both definitions coincide. However, this is in general not true. For a minimally coupled scalar field one has, for example,

$$
c_{w}^{2}=-1+\frac{2 \varepsilon}{3}-\frac{\eta}{3}, \quad c_{s}^{2}=1,
$$

with $\varepsilon, \eta$ the usual slow-roll parameters. In this sense, the second definition is more general: It can apply both to a fluid and to a scalar field, hence should be regarded as the proper definition of adiabaticity. Therefore we focus on the perturbation which satisfies $\delta P_{c, n a d}=0$ in this paper. As a consequence, for the first definition we then have (in agreement with [7])

$$
\delta P_{\text {nad }}=\left(c_{s}^{2}-c_{w}^{2}\right) \delta \rho_{c} .
$$

The third definition which is commonly used in the inflationary cosmology is about the stage when the so-called growing mode

\section{Formulas for arbitrary matter independent of gravity}

The conservation of the energy-momentum tensor in the comoving gauge gives $\delta\left(\nabla_{\mu} T_{j}^{\mu}\right)=0$.

$$
\delta P_{c}=-(\rho+P) A_{c} .
$$

Note that this relation between $\delta P_{c}$ and $A_{c}$ is completely independent of the theory of gravity. 


\section{Useful relations among gauge-invariant variables independent of gravity}

Combining Eqs. (1.3), (1.6) and (2.1), we now have

$$
\delta P_{\text {nad }}=\left(c_{s}^{2}-c_{w}^{2}\right) \delta \rho_{c}=\frac{c_{w}^{2}-c_{s}^{2}}{c_{s}^{2}}(\rho+P) A_{c} .
$$

The first equality is an identity, while the second comes from the conservation of the energy momentum tensor, and is valid for any gravity theory. This equation may be regarded as a statement that $\delta P_{\text {nad }}$ has the same behavior as $\delta \rho_{c}$ and $A_{c}$ unless $c_{w}^{2}=c_{s}^{2}$. In other words, the proper-time slicing $(A=0)$, comoving slicing $(v-B=0)$ and uniform density slicing $(\delta \rho=0)$ coincide with each other (approximately) if $c_{w}^{2} \neq c_{s}^{2}$ and $\delta P_{\text {nad }}=0$ (approximately). Namely,

$$
\left\{\delta \mathscr{P}_{\text {nad }} \approx 0, c_{s} \neq c_{w}\right\} \Rightarrow \delta \rho_{c} \approx A_{c} \approx 0 .
$$

We can use Eq. (3.1) to obtain for example a general relation between the comoving curvature perturbation $\mathscr{R}_{c}$ and uniform density curvature perturbation $\zeta$,

$$
\zeta=\mathscr{R}_{c}-\frac{H}{\dot{\rho}} \delta \rho_{c}=\mathscr{R}_{c}+\delta P_{n a d} \frac{H}{\dot{\rho}\left(c_{w}^{2}-c_{s}^{2}\right)}
$$

This is in agreement with the well-known coincidence of $\zeta$ and $\mathscr{R}_{c}$ on super-horizon scales for slow roll-models in general relativity, since in this case $c_{s} \neq c_{w}$ and $\delta P_{\text {nad }} \approx 0$ on superhorizon scales. Note also that this relation is degenerate in the case of $c_{s}=c_{w}$. As an example of such a case during inflation, later we explicitly consider the so-called ultra-slow roll inflation model.

\section{Discussion and conclusions}

The seminal works $[4,5]$ have taught us that for any relativistic theory of gravity, adiabaticity implies that $\zeta$ and $\mathscr{R}_{c}$ coincide and are conserved when gradient terms can be neglected, which in general happens on superhorizon scales. In this work, we have provided more insight into this claim.

First, we have specified that the above statement holds when (non)-adiabaticity is defined in the thermodynamical sense, see Eq. (1.2). We have argued that for a system out of equilibrium, like the early universe, one should define (non)-adiabaticity in the strict sense, as in Eq. (1.4). In this work, we have looked at perturbations which are strictly adiabatic in that strict sense $\left(\delta P_{c, \text { nad }}=0\right)$, and checked the implications for non-adiabaticity in the thermodynamical sense $\delta P_{\text {nad }}$. A third definition of non-adiabaticity states that the adiabatic limit has been reached as soon as the timedependent solution (the non-freezing one) for $\zeta$ has become totally negligible.

Second, we have rewritten the relation between (thermodynamical) non-adiabaticity and conserved quantities in such a way as to clarify when exactly gradient terms can be neglected, bypassing the need for an explicit computation of these gradient terms. In Eq. (3.1) we have shown that for any gravity theory, $\delta P_{n a d}$ is proportional to the lapse function in comoving slicing, $A_{c}$, provided that $c_{s}^{2} \neq c_{w}^{2}$. In the particular case of general relativity, $A_{c}$ is proportional to $\dot{\mathscr{R}}_{c}$ so we obtain the proportionality between $\delta P_{\text {nad }}$ and $\dot{\mathscr{R}}_{c}$, still under the condition that $c_{s}^{2} \neq c_{w}^{2}$. Furthermore, we have obtained in Eq. (3.3) that when $\delta P_{\text {nad }}=0, \mathscr{R}_{c}$ and $\zeta$ coincide, again under the condition that $c_{s}^{2} \neq c_{w}^{2}$. This results holds independently of gravity theory as well. 


\begin{tabular}{|l|l|l|}
\hline & Any Gravity theory & General Relativity $\left(A_{c}=\dot{\mathscr{R}}_{c} / H\right)$ \\
\hline Generic matter & $\delta P_{\text {nad }}=\delta \rho_{c}\left(c_{s}^{2}-c_{w}^{2}\right)=\left[\left(\frac{c_{w}}{c_{s}}\right)^{2}-1\right](\rho+P) A_{c}$ & $\delta P_{\text {nad }}=\left[\left(\frac{c_{w}}{c_{s}}\right)^{2}-1\right](\rho+P) \frac{\mathscr{R}_{c}}{H}$ \\
\hline M. c. scalar field & $\delta P_{\text {nad }}=\left(c_{w}^{2}-1\right) A_{c} \dot{\phi}^{2}$ & $\delta P_{\text {nad }}=\left(c_{w}^{2}-1\right) \frac{\mathscr{R}_{c}}{H} \dot{\phi}^{2}$ \\
\hline
\end{tabular}

\begin{tabular}{|l|l|l|}
\hline & Any Gravity theory & General Relativity \\
\hline Generic matter & $\zeta=\mathscr{R}_{c}-\delta P_{\text {nad }} \frac{H}{\dot{\rho}\left(c_{s}^{2}-c_{w}^{2}\right)}=\mathscr{R}_{c}+\frac{H}{\dot{\rho}} \frac{\rho+P}{c_{s}^{2}} A_{c}$ & $\zeta=\mathscr{R}_{c}+(\rho+P) \frac{\mathscr{R}_{c}}{c_{s}^{2} H} \frac{H}{\dot{\rho}}$ \\
\hline M. c. scalar field & $\zeta=\mathscr{R}_{c}+A_{c} \dot{\phi}^{2} \frac{H}{\dot{\rho}}$ & $\zeta=\mathscr{R}_{c}+\dot{\phi}^{2} \frac{\mathscr{R}_{c}}{H} \frac{H}{\dot{\rho}}$ \\
\hline
\end{tabular}

Table 1: The upper table shows the relation between the fluid-based non-adiabatic pressure perturbations $\delta P_{\text {nad }}$ and metric perturbations, and the lower table gives the relation between curvature perturbations on uniform density slices $\zeta$ and on comoving slices $\mathscr{R}_{c}$. For both tables the first column corresponds to relations valid in any gravity theory, the second column to the case of general relativity, the first row is for a generic matter field and the second one is for a minimally coupled scalar field.

Acknowledgments This work was supported by the Dedicacion exclusica and Sostenibilidad programs at UDEA, the UDEA CODI projects IN10219CE and 2015-4044 (AER).

\section{References}

[1] A. D. Linde, Phys. Lett. B 108, 389 (1982).

[2] A. Albrecht and P. J. Steinhardt, Phys. Rev. Lett. 48, 1220 (1982).

[3] A. A. Starobinsky, Phys. Lett. B 117, 175 (1982).

[4] D. Wands, K. A. Malik, D. H. Lyth and A. R. Liddle, Phys. Rev. D 62, 043527 (2000) [astro-ph/0003278].

[5] D. H. Lyth, K. A. Malik and M. Sasaki, JCAP 0505, 004 (2005) [astro-ph/0411220].

[6] A. J. Christopherson and K. A. Malik, Phys. Lett. B 675, 159 (2009) [arXiv:0809.3518 [astro-ph]].

[7] A. J. Christopherson, J. C. Hidalgo and K. A. Malik, JCAP 1301, 002 (2013) [arXiv:1207.1870 [astro-ph.CO]].

[8] N. C. Tsamis and R. P. Woodard, Phys. Rev. D 69, 084005 (2004) [astro-ph/0307463].

[9] W. H. Kinney, Phys. Rev. D 72, 023515 (2005) [gr-qc/0503017].

[10] M. H. Namjoo, H. Firouzjahi and M. Sasaki, Europhys. Lett. 101, 39001 (2013) [arXiv:1210.3692 [astro-ph.CO]].

[11] X. Chen, H. Firouzjahi, M. H. Namjoo and M. Sasaki, Europhys. Lett. 102, 59001 (2013) [arXiv:1301.5699 [hep-th]].

[12] X. Chen, H. Firouzjahi, E. Komatsu, M. H. Namjoo and M. Sasaki, JCAP 1312, 039 (2013) [arXiv:1308.5341 [astro-ph.CO]].

[13] S. Mooij and G. A. Palma, JCAP 11, 025 (2015) [arXiv:1502.03458 [astro-ph.CO]].

[14] J. Martin, H. Motohashi and T. Suyama, Phys. Rev. D 87, 023514 (2013) [arXiv:1211.0083 [astro-ph.CO]]. 